\title{
Identification of long non-coding RNAs expressed during the osteogenic differentiation of human bone marrow-derived mesenchymal stem cells obtained from patients with ONFH
}

\author{
TAO LI $^{1}$, KE XIAO $^{2}$, YINGXING XU $^{1}$, YUANZHONG REN ${ }^{1}$, YINGZHEN WANG ${ }^{1}$, \\ HAINING ZHANG ${ }^{1}$, XISHENG WENG ${ }^{3}$ and YAPING JIANG ${ }^{4}$
}

\author{
${ }^{1}$ Department of Joint Surgery, The Affiliated Hospital of Qingdao University, Qingdao, Shandong 266003; \\ ${ }^{2}$ Department of Orthopedic Surgery, West China Hospital, Sichuan University, Chengdu, Sichuan 610041; \\ ${ }^{3}$ Department of Orthopedic Surgery, Peking Union Medical College Hospital, Peking Union Medical College, Beijing 100730; \\ ${ }^{4}$ Department of Oral Implantology, The Affiliated Hospital of Qingdao University, Qingdao, Shandong 266003, P.R. China
}

Received May 9, 2020; Accepted August 11, 2020

DOI: $10.3892 /$ ijmm.2020.4717

\begin{abstract}
Long non-coding RNAs (lncRNAs) are crucial for the occurrence and development of numerous diseases. Although lncRNAs are involved in the biological activities of stem cells and play crucial roles in stem cell differentiation, the expression of specific lncRNAs during human bone marrow-derived mesenchymal stem cell (hBMSC) osteogenic differentiation in osteonecrosis of the femoral head (ONFH) and their regulatory roles have not yet been fully elucidated. To the best of our knowledge, the present study is the first to characterize lncRNA expression profiles during hBMSC osteogenic differentiation in ONFH using microarray analysis and RT-qPCR to confirm the microarray data. A total of 24 downregulated and 24 upregulated lncRNAs were identified and the results of RT-qPCR were found to be consistent with those of microarray analysis. Bioinformatics analyses, using the Gene Ontology (GO) and Kyoto Encyclopedia of Genes and Genomes (KEGG) databases, were conducted to explore the possible mechanisms and identify the signaling pathways that the lncRNAs are involved in. GO analysis revealed significant changes in the intracellular organelle, Ras protein signal transduction and transferase activity. KEGG pathway analysis revealed that the lncRNAs were closely associated with fatty acid metabolism, apoptosis and the TGF- $\beta$ signaling pathway. The overexpression of MAPT antisense RNA 1 (MAPT-AS1) was found to promote osteogenesis and inhibit the adipogenesis of hBMSCs at the cellular and mRNA levels. On the whole, the findings of the present study identified the lncRNAs and their roles in hBMSCs undergoing osteogenic differentiation in $\mathrm{ONFH}$ and provide a new perspective for the pathogenesis of ONFH.
\end{abstract}

Correspondence to: Dr Yaping Jiang, Department of Oral Implantology, The Affiliated Hospital of Qingdao University, 59 Haier Road, Qingdao, Shandong 266003, P.R. China

E-mail: jyp6817@163.com

Key words: osteonecrosis of the femoral head, osteogenic differentiation, marrow-derived stem cells, lncRNA profile

\section{Introduction}

As a pathological state with multiple possible etiologies, osteonecrosis of the femoral head (ONFH), also known as avascular necrosis, results in decreased vascular supply to the subchondral bone of the femoral head, ultimately resulting in osteocyte death and the collapse of the articular surface (1). Several studies have examined the pathogenesis of ONFH, which has been demonstrated to involve the apoptosis of osteoblasts and osteocytes $(2,3)$, adipogenesis (4), venous congestion $(5,6)$ and mutations in the COL2A1 gene (7). However, the specific mechanisms underlying the pathology of ONFH remain poorly understood.

The potential of muscle, cartilage, bone and adipose tissue differentiation has resulted in the use of human bone marrow-derived mesenchymal stem cells (hBMSCs) (8) therapeutically in a clinical setting (9-13). As a key function of hBMSCs, osteogenic differentiation plays a crucial role in the formation and remodeling of bone. Long non-coding RNAs (lncRNAs) are transcripts with a length of $>200$ nucleotides that do not code for any proteins. The critical roles of lncRNAs in various physiological and pathological processes have been proven (14-18). It has also been demonstrated that lncRNAs may also participate in hBMSC osteogenic differentiation (19). Moreover, the abnormal expression of lncRNAs may lead to the development of diseases due to variations in the osteogenic differentiation capacity of hBMSCs. However, the differential expression profiles of lncRNAs expressed in hBMSCs from patients with ONFH have not yet been fully elucidated. Therefore, the present study aimed to examine the role of lncRNAs expressed during the osteogenic differentiation of abnormal hBMSCs obtained from patients with ONFH.

\section{Materials and methods}

Cells and cell culture. Patients who had undergone total hip arthroplasty (THA) due to femoral neck fracture or ONFH were included in the present study, whereas patients who had undergone THA for rheumatoid arthritis, ankylosing spondylitis and other diseases were excluded. In total, 3 patients who 
had undergone THA for the treatment of femoral neck fracture provided normal bone marrow tissue: All 3 were Chinese; 2 were females, aged 60 and 67 years, while the other patient was a 66-year-old male. ONFH bone marrow tissue was also obtained from another 3 patients, of which 2 were females, aged 55 and 61 years and the other patient was a 58-year-old male, on whom THA had been performed for ONFH. Bone marrow tissue was collected at the Affiliated Hospital of Qingdao University from January, 2018 to May, 2018. The femoral bone marrow tissue samples were used to extract the hBMSCs with density gradient separation, as previously described (20). Bone marrow diluted with an equal volume of PBS was layered over lymphocyte separate medium. The mononuclear cell layer was collected following centrifugation. The hBMSCs extracted were cultured in a stem cell medium in a humidified atmosphere with $5 \% \mathrm{CO}_{2}$ at $37^{\circ} \mathrm{C}$. The Ethics Committee of the Affiliated Hospital of Qingdao University approved the study, while informed consent was obtained from all participants.

Flow cytometric analysis. Surface antigen markers on the hBMSCs were detected using an Apogee A50-MICRO flow cytometer (Apogee Corporation). The hBMSCs were suspended in PBS at a concentration of approximately $10^{6}$ cells $/ \mathrm{ml}$ and washed twice with PBS. Approximately $5 \times 10^{5}$ cells per $500 \mu \mathrm{l}$ were incubated and stained with $5 \mathrm{ml}$ of mouse anti-human CD34-fluorescein isothiocyanate (FITC) (560942), CD45-FITC (560976), CD73-FITC (561254) and CD90-FITC (561969) antibodies for $20 \mathrm{~min}$ at room temperature. All antibodies used were purchased from BD Biosciences.

Osteogenic differentiation of hBMSCs. The hBMSCs (passage 3) were plated in growth medium in 6-well plates. When $80 \%$ confluency was reached, mesenchymal stem cell osteogenic differentiation medium was used as the growth medium. The medium was changed every 3 days. The RT-qPCR analysis of osteogenic differentiation markers [alkaline phosphatase (ALP), Runt-related transcription factor 2 (RUNX2), osteopontin (OPN) and bone sialoprotein (BSP)] and staining (ALP staining and Alizarin Red staining) were adopted to detect the osteogenic ability of the hBMSCs. All experiments were performed in triplicate.

$R A N$ extraction and $R T-q P C R$. Total RNA extraction was performed form the hBMSCs using TRIzol reagent (Invitrogen; Thermo Fisher Scientific, Inc.), following instructions provided by the manufacturer. The expression of osteogenesis- and adipogenesis-related genes, including OPN, BSP, Runx2, ALP and peroxisome proliferator-activated receptor $\gamma$ (PPAR $\gamma)$ were determined by RT-qPCR. Total RNA was reverse transcribed using oligo-dT primers. The cDNA was utilized as a template to amplify target genes with the SYBR Premix Ex Tag kit (Takara Bio, Inc.). The primers of these genes are listed in Table I. Each RNA sample was evaluated in triplicate and PCR cycles were as follows: $94^{\circ} \mathrm{C}$ for $5 \mathrm{~min}, 95^{\circ} \mathrm{C}$ for $30 \mathrm{sec}$, $58^{\circ} \mathrm{C}$ for $30 \mathrm{sec}$, and $72^{\circ} \mathrm{C}$ for $30 \mathrm{sec}(35$ cycles $), 94^{\circ} \mathrm{C}$ for $5 \mathrm{~min}$. Relative expression of mRNA was evaluated using the $2^{-\Delta \Delta \mathrm{Cq}}$ method and normalized to the expression of GAPDH (21).

Staining. Alizarin Red and ALP staining were adopted to evaluate the osteogenic differentiation capacity of the hBMSCs. An
ALP staining kit (Tianjin Blood Research Institute) was used on day 3, as instructed by the manufacturer, to conduct ALP staining. The cells are processed according to the following procedures: No. 1 solution was added at room temperature for $1 \mathrm{~min}$, followed by rinsing for $2 \mathrm{~min}$. The staining solution was then added followed by incubation at $37^{\circ} \mathrm{C}$ for $2 \mathrm{~h}$ and rinsing for $2 \mathrm{~min}$. No. 5 solution was then added for re-staining for $5 \mathrm{~min}$, followed by rinsing for $2 \mathrm{~min}$, and drying. For Alizarin Red staining, after washing the cells twice with PBS, fixing was performed using $95 \%$ ethanol for $20 \mathrm{~min}$, and the cells were washed 3 times using distilled water, and were stained using Alizarin Red solution (Sigma-Aldrich; Merck KGaA) for $30 \mathrm{~min}$ at $37^{\circ} \mathrm{C}$. Oil Red O staining was performed to evaluate the adipogenic differentiation capacity of the hBMSCs. Cells were washed twice with PBS and fixed with $10 \%$ formalin for $10 \mathrm{~min}$ at room temperature. After fixation, cells were stained with filtered Oil Red O solution (Sigma-Aldrich; Merck KGaA) for $1 \mathrm{~h}$ at room temperature.

Microarray analysis. Three normal cell samples of osteogenic differentiation were used as the controls, while 3 osteogenic differentiation samples obtained from patients with ONFH were used as the experimental group. TRIzol reagent (Invitrogen; Thermo Fisher Scientific, Inc.) was used to extract total RNA from the hBMSCs, while a mirVana miRNA Isolation kit (Ambeon; Thermo Fisher Scientific, Inc.) was used as instructed by the manufacturer for purification. Following RNA extraction, labeling, hybridization and amplification, the CapitalBiotech human array used 4 identical arrays for each slide to design the lncRNA Array V4.0, while each array contained probes that could interrogate approximately 41,000 human IncRNAs. The probes were used to detect each RNA and the process was repeated for confirmation. A total of 4,974 control probes (Agilent Technologies, Inc.) constituted the array. The analysis of the IncRNA array data was conducted using GeneSpring software V13.0 (Agilent Technologies, Inc.) for data summarization, normalization and quality control. The differentially expressed genes were obtained using a t-test P-value of 0.05 and a fold change of $\geq 2$ and $\leq-2$ as the threshold values. The adjust data function of CLUSTER 3.0 software was applied for $\log 2$ transformation of the data and median centering by the genes, while further analysis was conducted using hierarchical clustering with an average linkage.

Using RT-qPCR, the identity of 6 lncRNAs [AC107070.1, linc-ANKRD20A1-4, RP11-794G24.1, long intergenic non-protein coding RNA 473 (LINC00473), MAPT antisense RNA 1 (MAPT-AS1) and AP005273.1] was further confirmed. The primers used for the lncRNAs are listed in Table I.

Furthermore, hBMSCs were islated from another 30 samples, including 15 normal and 15 patients with ONFH who underwent THA in the Department of Joint surgery at The Affiliated Hospital of Qingdao University from October, 2018 to September, 2019. Informed consent form was obtained from all participants. The expression levels of 2 upregulated lncRNAs (AC107070.1 and linc-ANKRD20A1-4) and 2 downregulated lncRNAs (LINC00473 and MAPT-AS1) were examined.

Target gene prediction. In the present study, the functions of cis and trans target mRNAs were used to predict the target 
Table I. Primers of lncRNAs and the related osteogenic genes.

Gene

Primer sequence 5'-3'

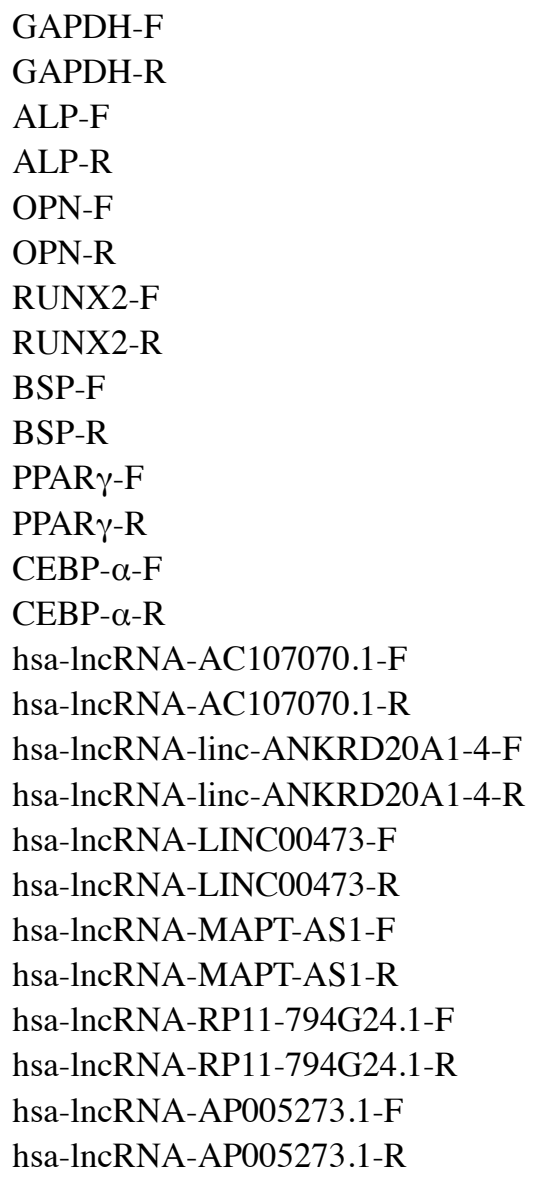

GGTCACCAGGGCTGCTTTTA

GGATCTCGCTCCTGGAAGATG

CCACGTCTTCACATTTGGTG

AGACTGCGCCTGGTAGTTGT

ACTCGAACGACTCTGATGATGT

GTCAGGTCTGCGAAACTTCTTA

TGTCATGGCGGGTAACGAT

AAGACGGTTATGGTCAAGGTGAA

TGGATGAAAACGAACAAGGCA

AAACCCACCATTTGGAGAGGT

CCTATTGACCCAGAAAGCGATT

CATTACGGAGAGATCCACGGA

AGGAACACGAAGCACGATCAG

CGCACATTCACATTGCACAA

CACATTCCAGCCAAGGTAG

CAGCCTCTCAGACCACATTC

TGGAGTTGGACATTTGTGG

TGGAGTTGGACATTTGTGG

GAGGTCTGAGTCCGAAGTTG

AGCAGGCAGATTCCAAAG

TCCGCTGGAAAGAGAACTC

CCTGTGAGGGCATACACC

GGCGTGGATCTTGGAGAGTC

GATGCTGGACGAATCCCAGT

TTCTTGACCCTCTCCAATGTGA

ACTGTCCAATAGCTTCCATCAGG

genes of the lncRNAs. Protein-coding genes within a $100 \mathrm{~kb}$ genomic distance from the lncRNA were defined as potentially cis-regulated target genes, and protein-coding genes co-expressed with the IncRNA with a Pearson's correlation coefficient ( $|r|>0.95$ ) and a $>100 \mathrm{~kb}$ genomic distance from the IncRNA or in different chromosomes were defined as potentially trans-regulated target genes.

Lentivirus vector construction and infection of hBMSCs for MAPT-AS1. The pRLenti-EF1a-EGFP-CMV-MAP T-AS1-overexpression lentivirus (H12785) was obtained from OBiO Technology. The hBMSCs were infected with MAPT-AS1-overexpression lentivirus at a final multiplicity of infection (MOI) of 100 containing $5 \mu \mathrm{g} / \mathrm{ml}$ polybrene, and observed for the expression of green fluorescent protein (GFP) using an inverted fluorescence microscope (EVOS FL, Invitrogen; Thermo Fisher Scientific, Inc.) after $24 \mathrm{~h}$. The transfection efficiency of MAPT-AS1 was determined by RT-qPCR after 3 days.

Bioinformatics analysis. Gene Ontology (GO) was utilized to identify the molecular functions of the differentially expressed genes. The GO category was also calculated. Furthermore, the differentially expressed lncRNAs were used in a pathway analysis that was performed using the latest Kyoto Encyclopedia of
Genes and Genomes (KEGG) database to analyze the potential functions of target genes.

Statistical analysis. All statistical analyses were conducted using SPSS statistical software v.16.0 (SPSS, Inc.). All data are expressed as the means \pm standard deviation. Comparisons between 2 variables of microarray data was performed using the Student's t-test. Comparisons between multiple groups were performed using the Kruskal-Wallis test along with Dunn's post hoc test. The $\chi^{2}$ test and Fisher's exact test were adopted for the GO and KEGG analyses. Statistical significance was considered to be indicated by P-values of $<0.05$.

\section{Results}

hBMSCs and osteogenic differentiation. The hBMSCs isolated from the bone marrow samples were spindle-shaped cells (Fig. 1) and no morphological differences were found between the 2 groups. ALP staining, Alizarin Red staining and osteogenic markers, including ALP, RUNX2, OPN and BSP, were used to detect the osteogenic ability of the hBMSCs. Positive ALP staining and Alizarin Red staining results revealed mineral deposits and bone formation (Fig. 1). The results of flow cytometric analysis are also shown in Fig. 1. The results 

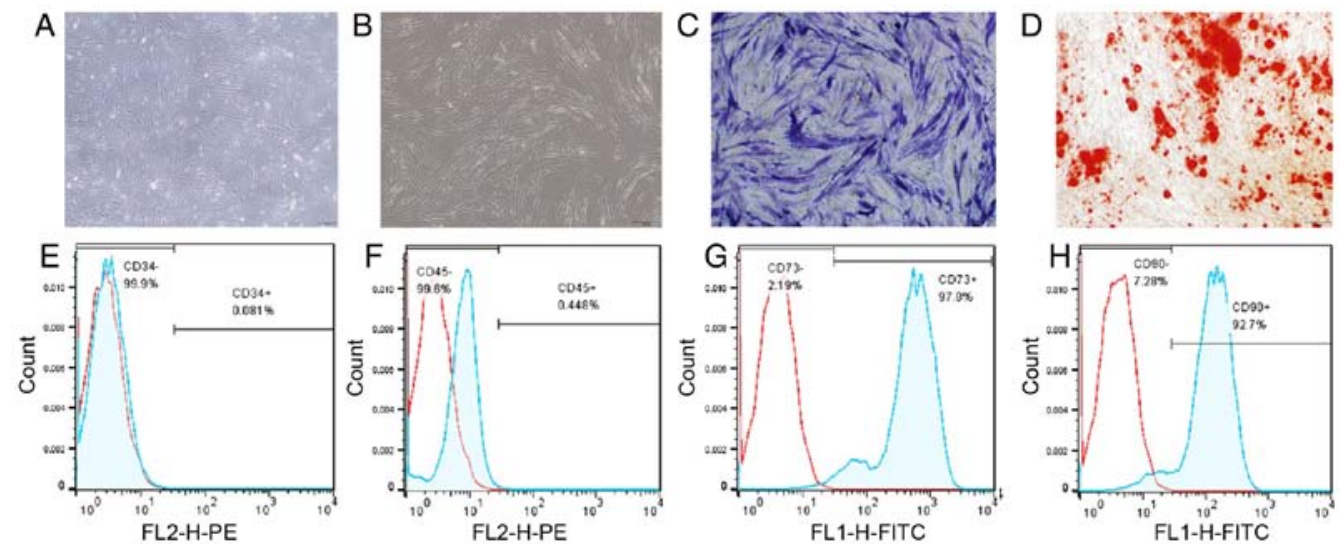

Figure 1. Morphology of the hBMSCs. (A) Normal hBMSCs cultured on day 6 were adherent, spindle and flat. (B) hBMSCs induced using osteogenic medium exhibited a swirl-like pattern. (C and D) Identification of osteogenic capacity by (C) positive ALP staining and (D) positive Alizarin Red staining. (E-H) Results of flow cytometric analysis. hBMSCs, human bone marrow-derived mesenchymal stem cells; ALP, alkaline phosphatase.
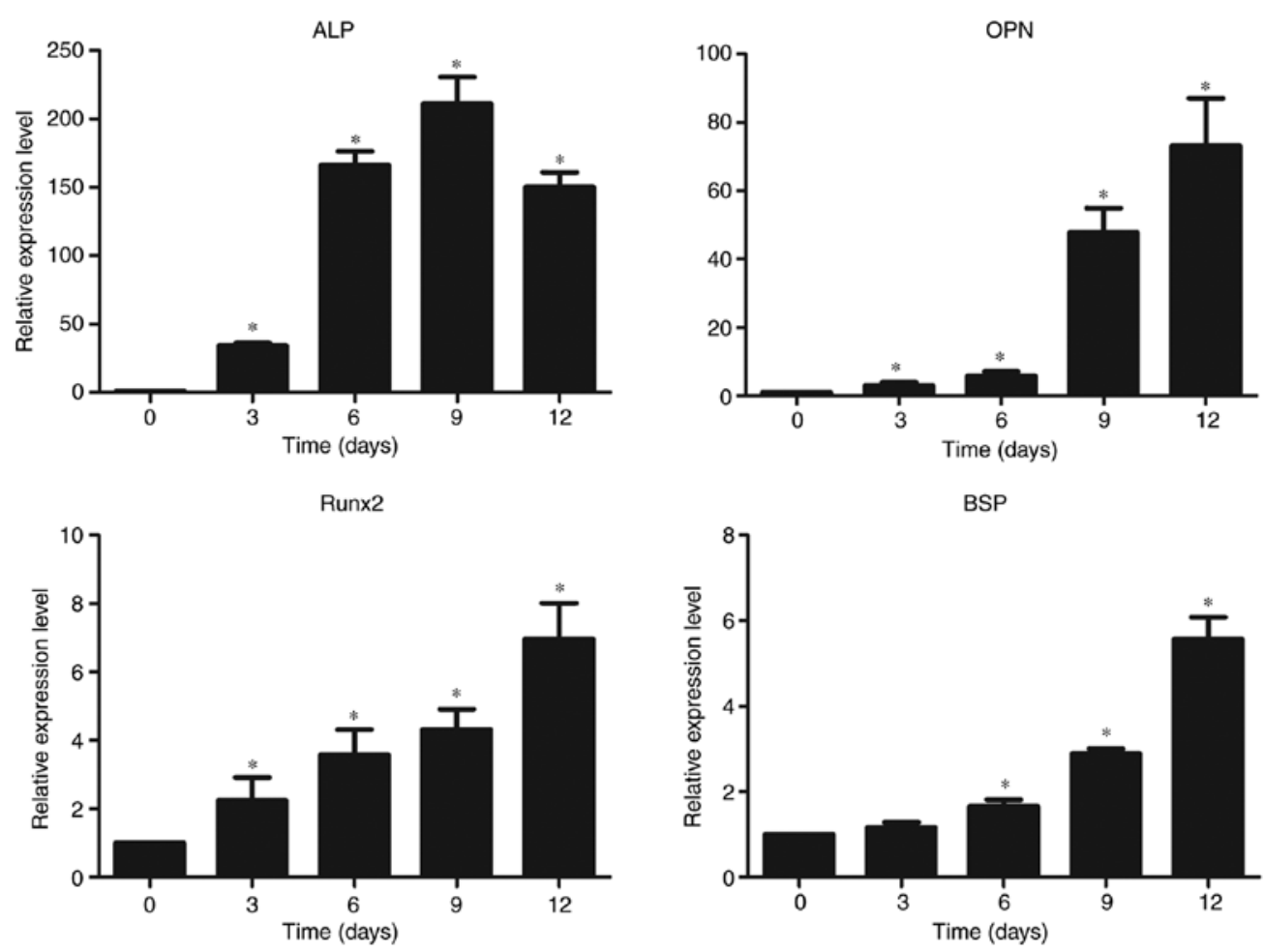

Figure 2. hBMSCs were confirmed to be undergoing osteogenic differentiation by RT-qPCR of the mRNA expression levels of ALP, OPN, RUNX2 and BSP. " $\mathrm{P}<0.05$ compared to day 0 . hBMSCs, human bone marrow-derived mesenchymal stem cells; ALP, alkaline phosphatase; OPN, osteopontin; RUNX2, runt-related transcription factor 2; BSP, bone salioprotein.

of RT-qPCR revealed the elevated expression levels of OPN, RUNX2, ALP and BSP (Fig. 2).

Expression profiles of lncRNAs in hBMSCs from patients with $O N F H$. IncRNA expression levels during hBMSC osteogenic differentiation were detected using lncRNA microarray chips (The CapitalBiotech human IncRNA Array V4.0). A total of 48 differentially expressed lncRNAs were identified, including 24 lncRNAs, which were upregulated (RP11-216N14.9, RP11-989E6.10, BX571672.1, RP11-230G5.2, linc-SLC30A5-5, BX004987.4, AC107070.1, RP11-265D17.2, RP11-1060J15.4, RP11-794G24.1, linc-ANKRD20A1-4, RP11-485O10.2, AC097532.2, RP11-143J24.1, RP11-762H8.3,
CTD-2015H3.2, linc-C17orf97-2, RP11-513M1.1, RP11-22H5.2, linc-ANKRD20A1-2, linc-OR4M2-5, RP11-262H14.1, linc-LOC389493-3 and linc-ATP6V1C2-3) and 24 lncRNAs, which were downregulated (AC104135.3, RP11-26M5.3, MAPT-AS1, AL589743.1, linc-SLITRK1-4, RP11-229P13.19, AP005273.1, RP11-406O23.2, AP003900.6, RP11-525J21.1, linc-PENK-1, linc-PENK-2, RP5-1102E8.3, linc-CSTB-3, CTD-2314B22.3, RP5-1148A21.3, LINC00473, CTC-498M16.2, RP11-16M8.2, AP000525.9, linc-TCF4-3, RP11-645N11.2, RP11-17A4.2 and RP11-324O2.3). The differential expression of the 48 lncRNAs is presented in brief in Table II. Hierarchical clustering analysis revealed the expression profiles of the IncRNAs during the osteogenic 


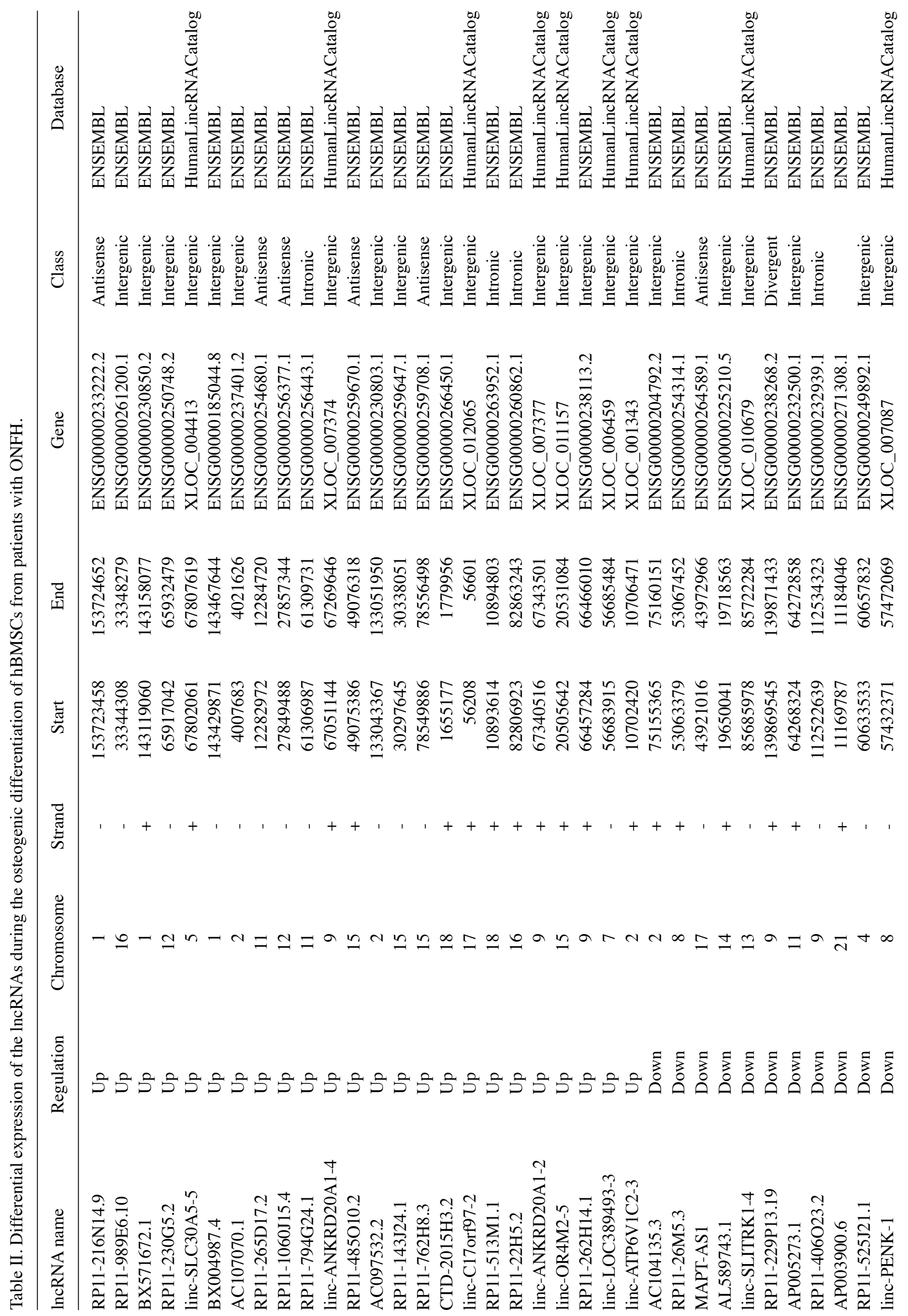




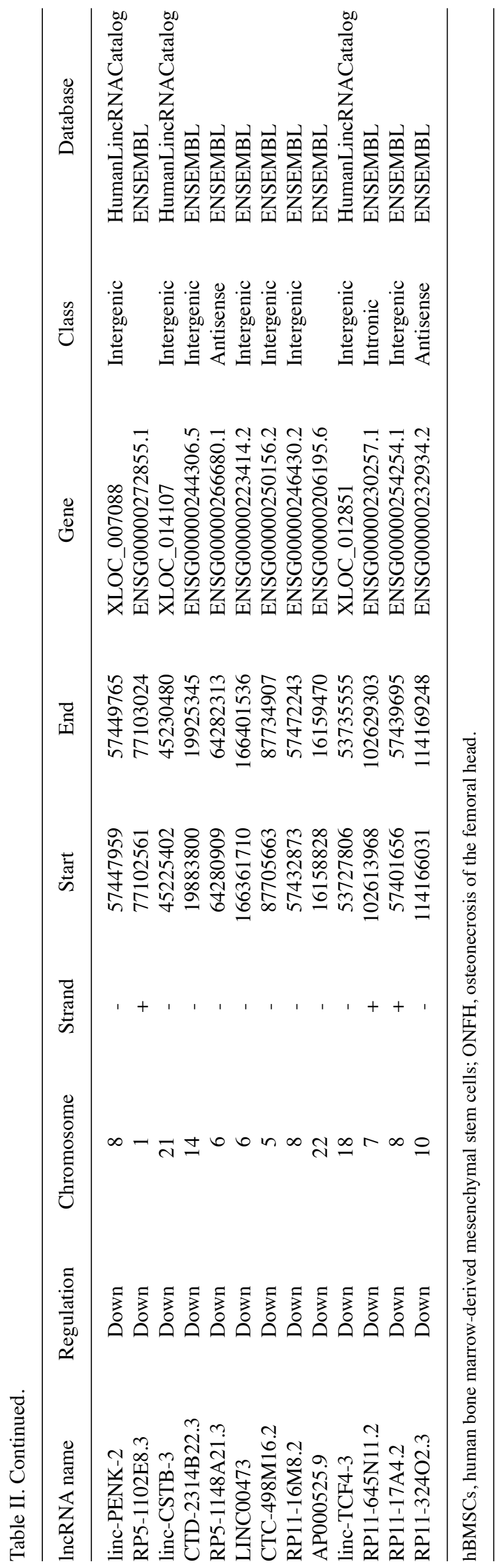

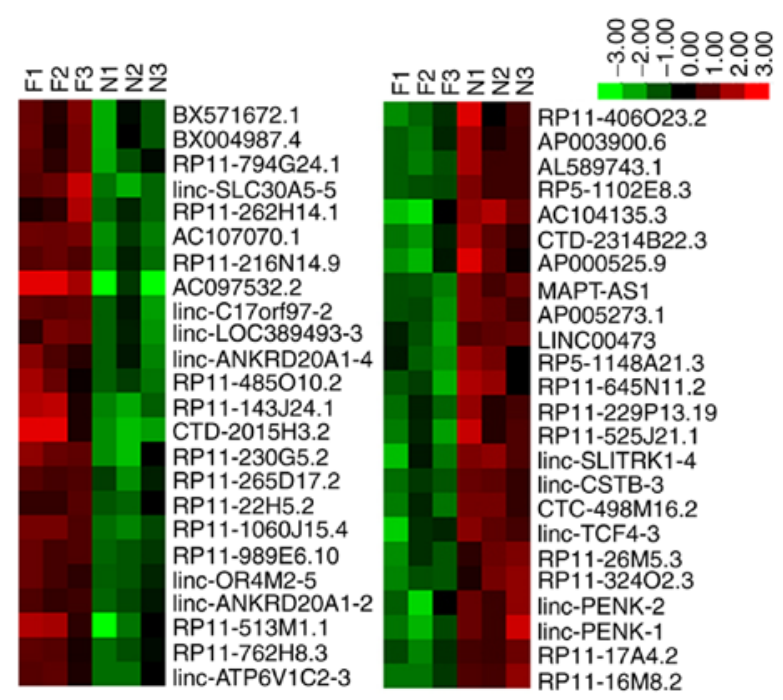

Figure 3. Heatmap based on the differential expression of the lncRNAs between the control group and the experiment group (control group, normal hBMSC samples obtained during osteogenic differentiation; experiment group, hBMSC samples obtained during osteogenic differentiation from patients with ONFH). hBMSCs, human bone marrow-derived mesenchymal stem cells; ONFH, osteonecrosis of the femoral head.

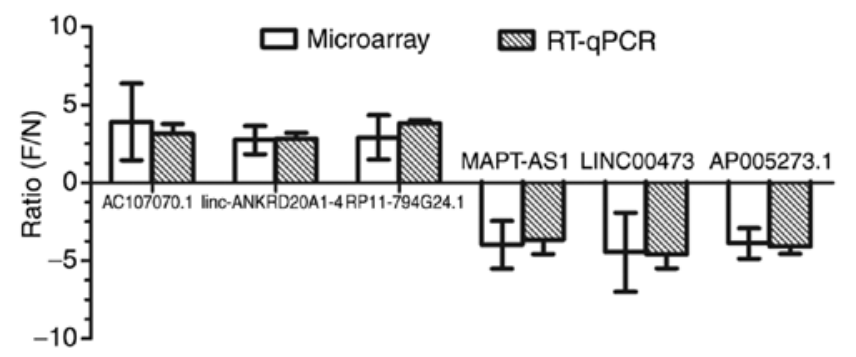

Figure 4. RT-qPCR was performed to confirm the identity of the upregulated lncRNAs (AC107070.1 and linc-ANKRD20A1-4) and downregulated lncRNAs (LINC00473 and MAPT-AS1) in the hBMSCs from patients with ONFH. hBMSCs, human bone marrow-derived mesenchymal stem cells; ONFH, osteonecrosis of the femoral head.

differentiation of hBMSCs from patients with ONFH and healthy subjects (Fig. 3).

Comparison between the RT-qPCR and microarray analyses. The microarray data analysis revealed 3 upregulated 1ncRNAs (AC107070.1, linc-ANKRD20A1-4 and RP11-794G24.1) and 3 downregulated lncRNAs (LINC00473, MAPT-AS1 and AP005273.1) which were selected for RT-qPCR analysis in the hBMSCs. The results of RT-qPCR revealed that the expression trends of these 4 lncRNAs were consistent with those of the microarray results, which are shown in Fig. 4.

The expression levels of AC107070.1, linc-ANKRD20A1-4, LINC00473 and MAPT-AS1 (Fig. 5) in the samples were also consistent with those of the results of the microarray analysis.

Target gene prediction and association study. In the present study, the genes involved were predicted based on the functional annotations of their related cis and trans target mRNAs. Further results are presented in detail in Table III. 

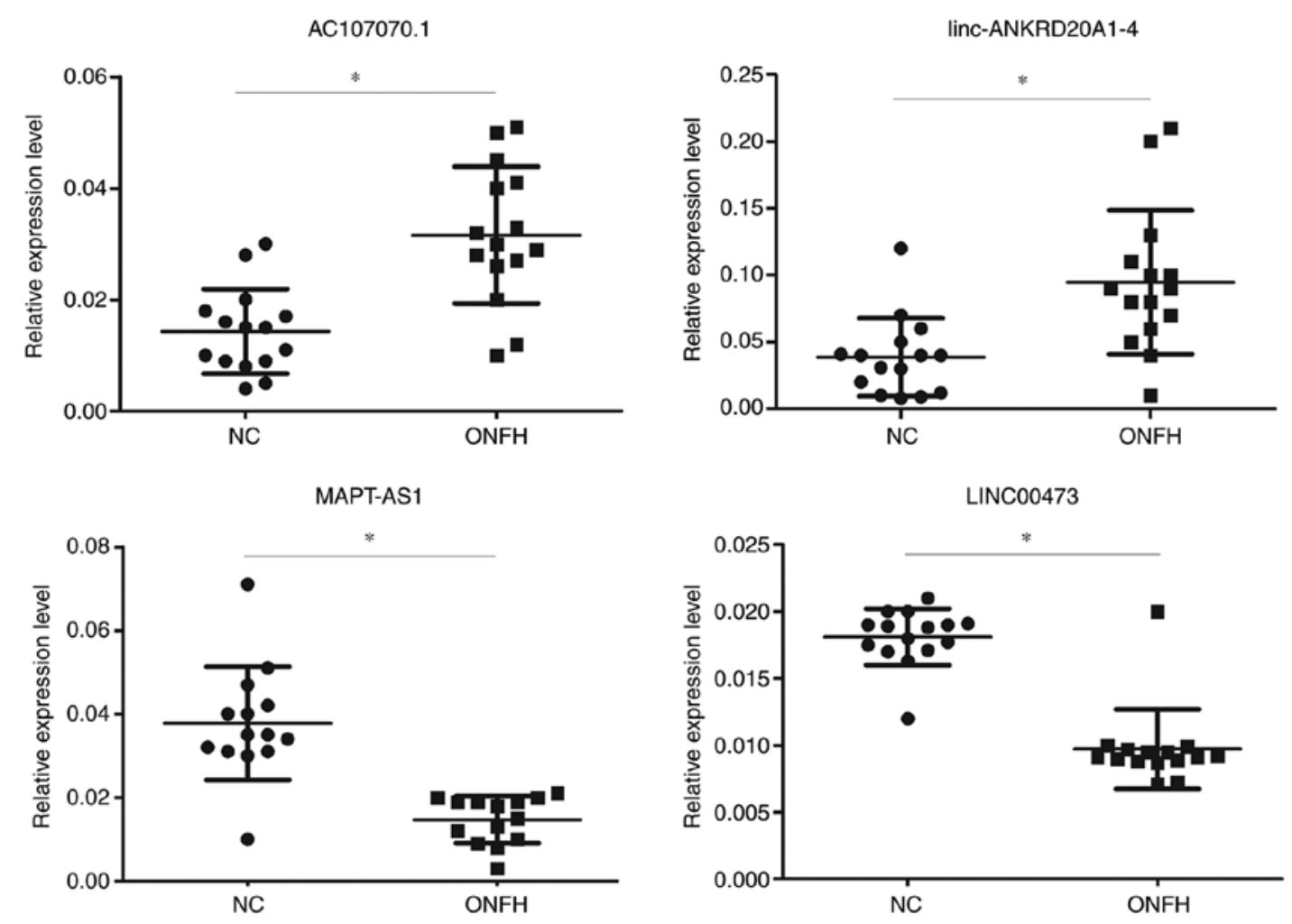

Figure 5. Expression levels of AC107070.1, linc-ANKRD20A1-4, LINC00473 and MAPT-AS1 in the samples. NC, normal control; ONFH, osteonecrosis of the femoral head.

Bioinformatics analysis of the DNA sequence. GO analysis mainly analyzes cellular components, biological processes and molecular functions. Cellular components involved were found to include the nucleolus, cytoplasm, RISC complex, small-subunit proteasome, endomembrane system, organelle membrane, intracellular part, cytoplasmic part, membrane-bounded organelle and intracellular organelle. Biological processes involved were found to include response to oxygen radical, fatty acid beta-oxidation, protein targeting to nucleus, small molecule catabolic process, cellular biosynthetic process, protein transport, cellular lipid metabolic process, metabolic process, intracellular transport and Ras protein signal transduction. Molecular functions involved were found to include ATP binding, lipid kinase activity, chemokine receptor activity, purine ribonucleoside triphosphate binding, purine ribonucleoside binding, oxidoreductase activity, oxidizing metal ions, NAD or NADP as acceptor, ATP-dependent microtubule motor activity, plus-end-directed, transferase activity, transferring sulfur-containing groups, sulfate transmembrane transporter activity and transferase activity. The results of GO analysis are presented in Fig. 6.

Pathway analysis was conducted using the KEGG database. It was found that several pathways (Wnt, VEGF, Notch, MAPK, hedgehog, NF- $\kappa \mathrm{B}$, calcium, FoxO, PPAR and TGF- $\beta$ signaling pathways, as well as mineral absorption, apoptosis and fatty acid metabolism) were involved in osteogenic differentiation in the 2 groups, as illustrated in Fig. 7.

MAPT-AS1 promotes osteogenesis and inhibits the adipogenesis of hBMSCs. MAPT-AS1, which was downregulated in hBMSCs from patients with ONFH during osteogenic differentiation, was selected for functional analysis and for the further verification of the findings. The results revealed that the overexpression of MAPT-AS1 significantly promoted osteogenic differentiation, as indicated by ALP staining for the mineralization and expression of the osteogenic transcription factors, Runx2 and BSP (Fig. 8). On the contrary, the upregulation of MAPT-AS1 inhibited adipogenic differentiation, as indicated by Oil Red $\mathrm{O}$ staining and the expression of the adipogenic transcription factors, CEBP- $\alpha$ and PPAR $\gamma$ (Fig. 8).

\section{Discussion}

ONFH occurs in young individuals aged 20-40 years and 15,000-20,000 new cases of femoral head necrosis are reported annually $(22,23)$. The causes of femoral head necrosis mainly include hormones, alcohol abuse and hip trauma. Among these, steroid-induced osteonecrosis of the femoral head (SONFH) accounts for $46.03 \%$ of total femoral head necrosis cases (23) and is the most common type of femoral head necrosis. For patients with early-stage ONFH, although early intervention can be performed through drug therapy, core decompression, interventional therapy, etc., their outcomes are not satisfactory. Approximately $65-85 \%$ of patients with femoral head necrosis will continue to develop the disease, leading to the collapse of the femoral head (24), resulting in the need for total hip replacement surgery. The majority of patients with ONFH are young adults, and the life of their prosthesis is limited; thus, they may require multiple revision surgeries in the future, which leads to a tremendous economic burden to the family and society. The aim of the present study was to explore the possible mechanisms of ONFH and provide a basis for further 
Table III. Predicted target genes of differentially expressed lncRNAs.

IncRNA $\quad$ Target gene

A, Partial lncRNAs overexpressed in hBMSCs during osteogenic differentiation in ONFH

\begin{tabular}{ll}
\hline RP11-216N14.9 & GATAD2B, ILF2, SLC27A3, CHTOP \\
RP11-989E6.10 & TP53TG3E \\
RP11-230G5.2 & HMGA2 \\
RP11-265D17.2 & MICAL2, PARVA, \\
RP11-1060J15.4 & MANSC4, MRPS35, KLHL42, \\
RP11-794G24.1 & TMEM138, DDB1, CYB561A3, PGA3, TKFC, \\
RP11-485O10.2 & GALK2, COPS2, SECISBP2L \\
AC097532.2 & NCKAP5 \\
RP11-143J24.1 & CHRFAM7A, GOLGA8R \\
RP11-762H8.3 & HYKK, CHRNA3, PSMA4, PSMA4, IREB2 \\
linc-C17orf97-2 & DOC2B, SCGB1C2 \\
RP11-513M1.1 & PIEZO2 \\
RP11-22H5.2 & CDH13 \\
linc-ANKRD20A1-2 & SPATA31A3 \\
linc-OR4M2-5 & GOLGA6L6 \\
linc-ATP6V1C2-3 & ATP6V1C2, NOL10, PDIA6,
\end{tabular}

B, Partial lncRNAs underexpressed in hBMSCs during osteogenic differentiation in ONFH

$\begin{array}{ll}\text { AC104135.3 } & \text { TACR1 } \\ \text { MAPT-AS1 } & \text { TMEM101, MPP2, CD300LG, LSM12, PYY } \\ \text { AL589743.1 } & \text { OR11H2, OR4Q3, OR4N2, OR4M1 } \\ \text { linc-SLITRK1-4 } & \text { SLITRK6 } \\ \text { AP005273.1 } & \text { NUDT22, GPR137, TRPT1, FKBP2, TRMT112, VEGFB } \\ \text { RP11-406O23.2 } & \text { KIAA1958, HSDL2 } \\ \text { RP5-1102E8.3 } & \text { PIGK, ST6GALNAC5 } \\ \text { linc-CSTB-3 } & \text { ADARB1, POFUT2, } \\ \text { CTD-2314B22.3 } & \text { OR4K1, OR4K14, OR4K2, OR4N2, OR4K15 } \\ \text { RP5-1148A21.3 } & \text { EYS } \\ \text { LINC00473 } & \text { ILF2, MMP2, USP9X, CHUK, STK11, RPS6KA2 } \\ \text { RP11-645N11.2 } & \text { RASA4, POLR2J3, SPDYE2, } \\ \text { RP11-324O2.3 } & \text { CCDC186, TDRD1, VWA2, }\end{array}$

hBMSCs, human bone marrow-derived mesenchymal stem cells; ONFH, osteonecrosis of the femoral head.

intervention treatment of early-stage ONFH. To the best of our knowledge, the present study is the first to describe the role of lncRNAs in hBMSCs during osteogenic differentiation in osteonecrosis of the femoral head and it has more practical clinical significance.

In recent years, scholars at home and abroad have conducted extensive research on the pathogenesis of ONFH and have proposed a multi-strand bone necrosis theory, including the intraosseous hypertension theory (25), coagulation mechanism change theory (26), lipid metabolism disorder theory (27), osteoporosis theory $(28,29)$, bone cell apoptosis theory (30), membrane particle theory (31), gene polymorphism (32) and immune factors (33). Additionally, the disease has been found to be associated with the proliferation, osteogenic and adipogenic differentiation of hBMSCs. For example, the proliferative capability of hBMSCs has been found to be inhibited in patients with ONFH compared with healthy individuals (34). miRNA-22 has been shown to inhibit the adipogenic differentiation of hBMSCs through the protein expression of HDAC6 (35), while miRNA-100 may target BMPR2, which leads to the inhibition of osteogenic differentiation of hBMSCs (36). However, the association between lncRNAs and the osteogenic differentiation of hBMSCs during the pathogenesis of ONFH remains unclear. To date, at least to the best of our knowledge, only one study conducted focused on lncRNAs involved in femoral head necrosis (37), and studies have not been conducted on the characteristics of the osteogenic differentiation of hBMSCs from patients with ONFH.

In the present study, differentially expressed lncRNAs during the osteogenic differentiation of hBMSCs in 


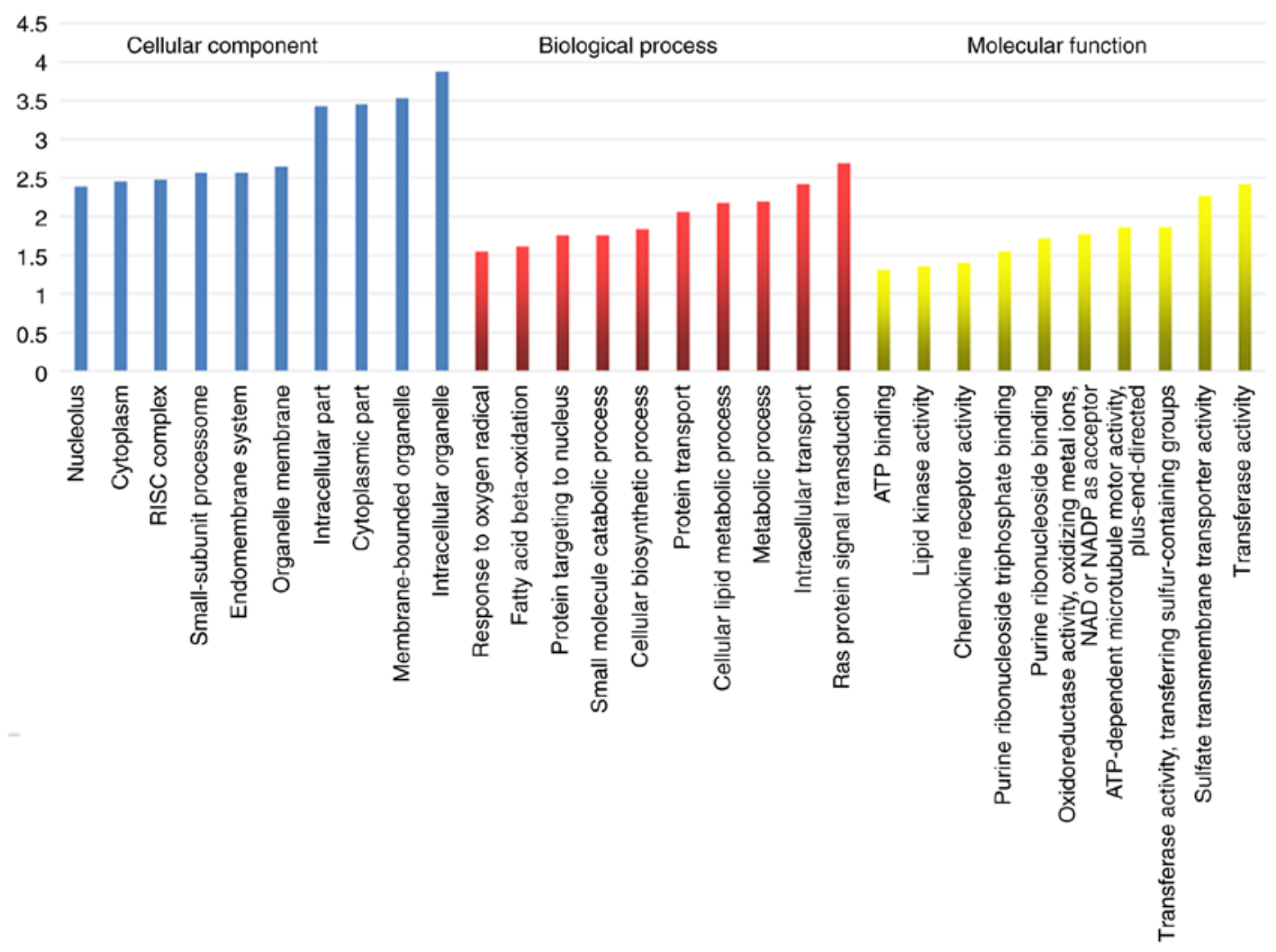

Figure 6. Results of the GO analysis, which identified the cellular components, biological processes and molecular functions involved.

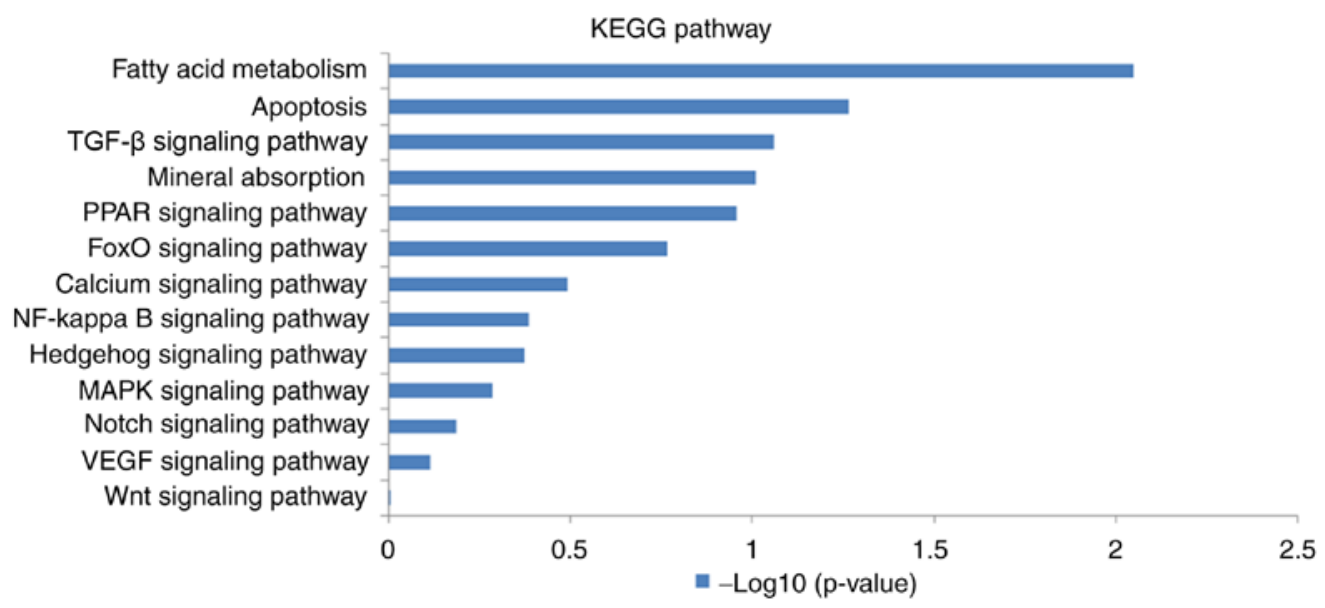

Figure 7. Results of the pathway analysis conducted using the KEGG database.

steroid-induced femoral head necrosis were identified using the CapitalBiotech human lncRNA Array V4.0. Bioinformatics analyses, including GO and pathway analysis of differentially expressed lncRNAs, were also conducted. CNC and ceRNA networks were also analyzed. The lncRNAs identified were further verified by RT-qPCR.

The expression levels of 24 downregulated and 24 upregulated lncRNAs were determined during the osteogenic differentiation of hBMSCs in ONFH. Targets of these lncRNAs were involved in processes, such as cell proliferation, differentiation and tumor metastasis. In total, 6 lncRNAs (AC107070.1, linc-ANKRD20A1-4, RP11-794G24.1, LINC00473, MAPT-AS1 and AP005273.1) in the hBMSCs were identified and confirmed by RT-qPCR. The results of RT-qPCR revealed that the expression trends of the 4 lncRNAs were consistent with those of the microarray analysis. Moreover, hBMSCs were isolated from another 30 samples, including 15 normal and 15 patients with ONFH. The expression levels of 2 upregulated lncRNAs (AC107070.1 and linc-ANKRD20A1-4) and 2 downregulated lncRNAs (LINC00473 and MAPT-AS1) were also consistent with those of the microarray and RT-qPCR analyses, which verified the accuracy of the results.

Since the majority of the lncRNAs in current databases have not yet been functionally annotated, their functions were predicted based on the functional annotations of their related 
A a

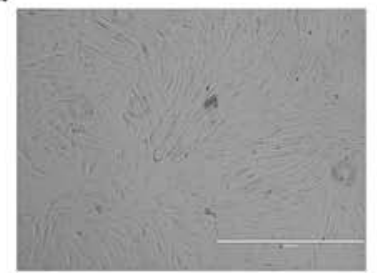

B a

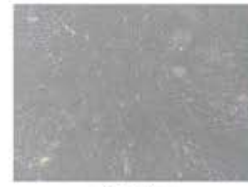

Control

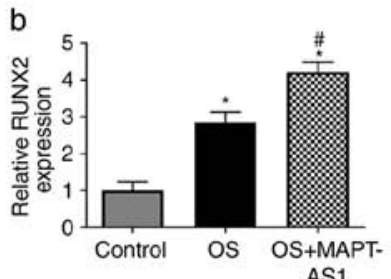

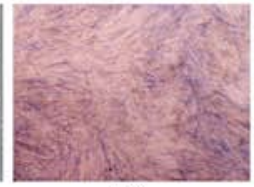

OS

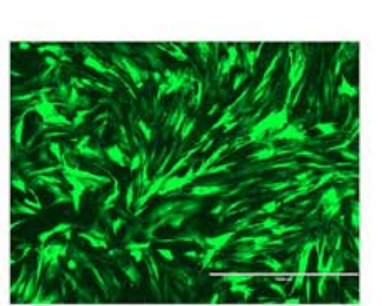

C a

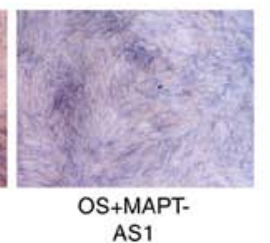

Control
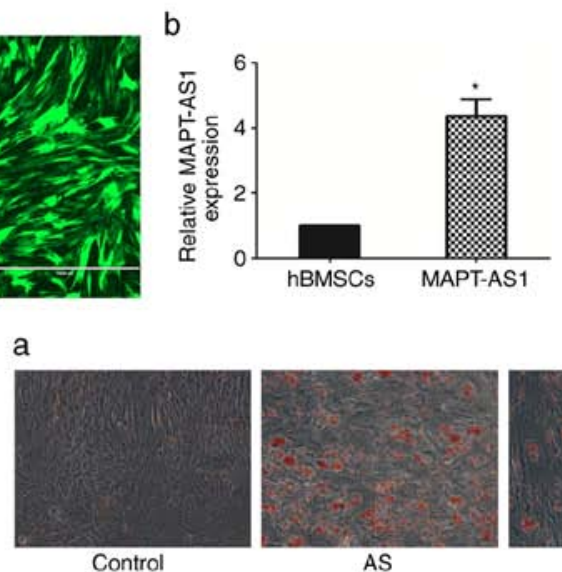

b
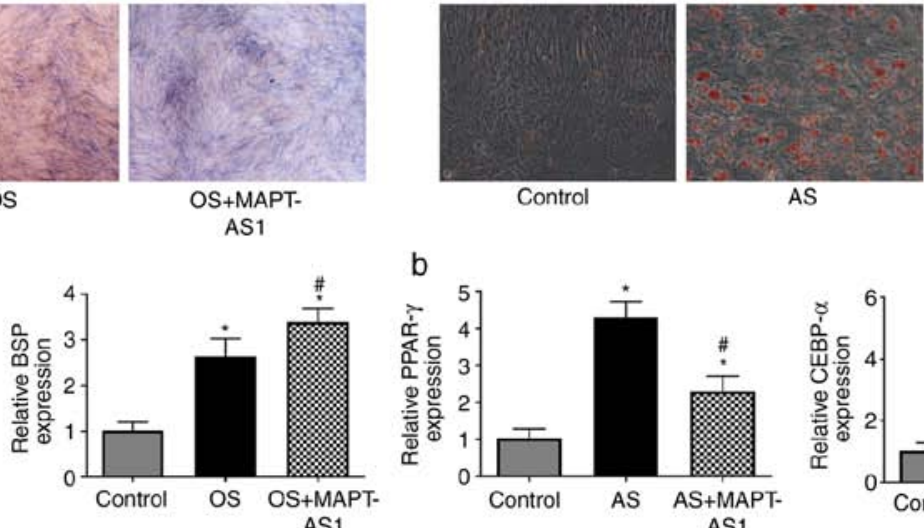

AS

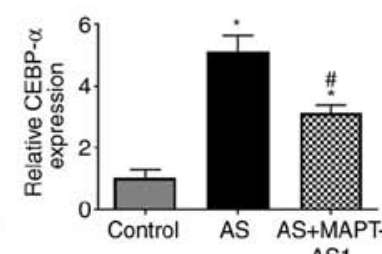

AS

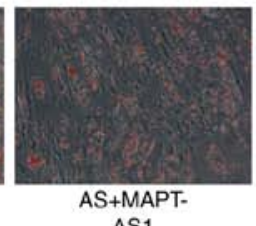

AS1

Figure 8. MAPT-AS1 promotes osteogenic differentiation and inhibits adipogenic differentiation of hBMSCs. (A-a) hBMSCs expressing GFP tagged MAPT-AS1-overexpression lentivirus under a fluorescence microscope; (A-b) transfection efficiency of MAPT-AS1 examined by RT-qPCR; (B-a) ALP staining; (B-b) mRNA expression levels of RUNX2 and BSP; (C-a) Oil red O staining; (C-b) mRNA expression levels of CEBP- $\alpha$ and PPAR $\gamma$. Control, normal hBMSC; OS, osteogenic differentiation of hBMSCs; OS + MAPT-AS1, osteogenic differentiation of overexpressed MAPT-AS1 hBMSCs; AS, adipogenic differentiation of hBMSCs; AS + MAPT-AS1, adipogenic differentiation of overexpressed MAPT-AS1 hBMSCs; hBMSCs, human bone marrow-derived mesenchymal stem cells. ${ }^{*} \mathrm{P}<0.05$, compared to control; ${ }^{*} \mathrm{P}<0.05$, compared to OS or AS.

cis and trans target mRNAs. From the information presented in Table III, it was found that one lncRNA can control multiple genes, such as RP11-794G24.1, RP11-762H8.3 and AP005273.1, while one gene can be regulated by several lncRNAs. For example, OR4N2 was found to be targeted by CTD-2314B22.3 and AL589743.1. The functions of these lncRNAs were also investigated using GO analysis to determine the biological processes, cellular components and molecular functions involved. Intracellular organelle, membrane-bounded organelle and cytoplasmic part were the 3 cellular components identified. Ras protein signal transduction, intracellular transport and metabolic process were the 3 biological processes identified. Moreover, the 3 most obvious aspects of change in molecular functions were found in transferase activity, sulfate transmembrane transporter activity and transferase activity, as well as transferring sulfur-containing groups. Fatty acid metabolism, apoptosis and TGF- $\beta$ signaling pathway were the 3 signaling pathways that exhibited the highest level of correlation in the KEGG pathways analysis. These 3 signaling pathways are inextricably linked to femoral head necrosis. For example, adipogenic overdifferentiation, osteoblast apoptosis and the inhibition of osteogenic differentiation through TGF- $\beta$ play vital roles in the occurrence and development of femoral head necrosis. However, carbon metabolism, the pentose phosphate pathway and biosynthesis of amino acids were significantly upregulated in the KEGG analysis (37). It was hypothesized that the difference in the results was due to two aspects. First, the method used differed. In the experiments in the present study, the hBMSCs underwent osteogenic differentiation prior to microarray analysis, while in the other study, the hBMSCs were screened using microarray analysis before undergoing osteogenic differentiation. Second, differences may also be due to individual differences in the cases included.

In the present study, LINC00473, also known as LNC473, C6orf176, bA142J11.1, and is located in the 6q27 region and LINC00473, was downregulated in the hBMSCs following osteogenic differentiation. It has been found that LINC00473 was involved in the development of a number of diseases including preeclampsia (38), colorectal cancer (39), gastric cancer (40) and others. It has been demonstrated that LINC00473 is involved in the pathogenesis and development of preeclampsia and may be a candidate biomarker, as well as a therapeutic target for preeclampsia (38). Wang et al found that LINC00473 promoted Taxol resistance via miR-15a in colorectal cancer (39). Zhang and Song demonstrated that LINC00473 is an IncRNA that is associated with prognosis and malignancy in gastric cancer, while it also regulates gastric cancer cell invasion and migration (40). In the present study, the expression of LINC00473 in ONFH was found to be lower than that in normal hBMSCs, and it was hypotehsized that LINC00473 plays an important role in the osteogenic and adipogenic differentiation of stem cells via related signaling pathways.

The MAPT-AS1 gene is located in the 17q21.31 region and in the present study, MAPT-AS1 was found to be downregulated during the osteogenic differentiation of hBMSCs. MAPT-AS1 is involved in the occurrence and development of tumors and Parkinson's disease. MAPT-AS1 overexpression has not been found in breast cancer; however, in triple-negative 
type (TNBC), a high MAPT-AS1 expression has been found to be associated with a longer patient survival (41). Additionally, MAPT-AS1 levels have been shown to be associated with MAPT expression, which is associated with breast cancer survival. The results of that study indicated that MAPT-AS1 may function as a potential breast cancer survival prediction biomarker (41). Pan et al (42) found that patients with ER-negative breast cancer who had larger tumors $(\geq 2 \mathrm{~cm})$, were of a younger age $(<60)$, were at stages (III-IV) and had metastatic lymph nodes, exhibited higher levels of MAPT-AS1 expression. The regulation of natural comparable sense MAPT transcripts in cells of ER-negative breast cancer leads to the association between MAPT-AS1 and paclitaxel resistance, invasiveness and cell growth. Research has indicated that overexpression may partially protect the MAPT mRNA from degradation by the overexpression of MAPT-AS1, while the knockdown of MAPT-AS1 decreases MAPT mRNA stability. Moreover, the knockdown of MAPT also decreases MAPT-AS1 mRNA expression. MAPT-AS1 expression is coordinated with that of MAPT in breast tumor tissues (42). Moreover, MAPT-AS1 and DNMT1 have been identified as potential epigenetic regulators of MAPT expression in Parkinson's disease across 4 different brain regions and an increased MAPT expression may be associated with the disease state, but not with the neuropathology severity of Parkinson's disease (43). In the present study, the overexpression of MAPT-AS1 significantly promoted the osteogenic differentiation and inhibited the adipogenic differentiation of hBMSCs at the cellular and mRNA level, as indicated by relevant staining and RT-qPCR analysis.

There were several limitations to the present study. First, the sample size of the present study was small, which may affect the results of the microarray analysis. Second, the majority of the IncRNAs require further validation by RT-qPCR. Third, the specific functions, as well as mechanisms of lncRNAs warrant further investigation.

In conclusion, to the best of our knowledge, this is the first study to elucidate the hBMSC expression profiles during osteogenic differentiation in ONFH. A total of 24 downregulated lncRNAs and 24 upregulated lncRNAs were found to be expressed during the osteogenic differentiation of hBMSCs from patients with ONFH. A bioinformatics analysis of the functions and mechanisms of the identified lncRNAs was conducted. The present study may provide a new perspective of the pathogenesis of ONFH and a novel direction for the early treatment of ONFH.

\section{Acknowledgements}

Not applicable.

\section{Funding}

The present study was supported by the National Natural Science Foundation of China (grant no. 81802151), the Shandong Province Natural Science Foundation (grant nos. ZR2016HQ05, no. ZR2017BH089 and ZR2019MH012), the China Postdoctoral Science Foundation (grant no. 2018M642616) and the Qingdao Applied Foundational Research Youth Project (grant no. 19-6-2-55-cg).

\section{Availability of data and materials}

The datasets used and/or analyzed during the current study are available from the corresponding author on reasonable request.

\section{Authors' contributions}

TL, YX, KX and YR performed the experiments and analyzed the results; YX, KX and YR wrote and drafted the manuscript; $\mathrm{HZ}$ and $\mathrm{XW}$ wrote, reviewed and edited the manuscript; $\mathrm{HZ}$ and YW conceived the methodology; while YJ and XW designed the research study and was a major contributor in recruiting the donors. All authors read and approved the final manuscript.

\section{Ethics approval and consent to participate}

The present study was approved by the Ethics Committee of the Affiliated Hospital of Qingdao University and written informed consent was obtained from all donors included in the study.

\section{Patient consent for publication}

Not applicable.

\section{Competing interests}

The authors declare that they have no competing interests.

\section{References}

1. Guerado E and Caso E: The physiopathology of avascular necrosis of the femoral head: An update. Injury 47 (Suppl 6): S16-S26, 2016.

2. Youm YS, Lee SY and Lee SH: Apoptosis in the osteonecrosis of the femoral head. Clin Orthop Surg 2: 250-225, 2010.

3. Calder JD, Buttery L, Revell PA, Pearse M and Polak JM: Apoptosis-a significant cause of bone cell death in osteonecrosis of the femoral head. J Bone Joint Surg Br 86: 1209-1213, 2004.

4. Assouline-Dayan Y, Chang C, Greenspan A, Shoenfeld Y and Gershwin ME: Pathogenesis and natural history of osteonecrosis. Semin Arthritis Rheum 32: 94-124, 2002.

5. Cui Q, Wang GJ, Su CC and Balian G: The otto aufranc award. Lovastatin prevents steroid induced adipogenesis and osteonecrosis. Clin Orthop Relat Res: 8-19, 1997.

6. Wang GJ, Sweet DE, Reger SI and Thompson RC: Fat-cell changes as a mechanism of avascular necrosis of the femoral head in cortisone-treated rabbits. J Bone Joint Surg Am 59: 729-35, 1977.

7. Kannu P, O'Rielly DD, Hyland JC and Kokko LA: Avascular necrosis of the femoral head due to a novel $\mathrm{C}$ propeptide mutation in COL2A1. Am J Med Genet A 155A: 1759-1762, 2011.

8. Pittenger MF, Mackay AM, Beck SC, Jaiswal RK, Douglas R, Mosca JD, Moorman MA, Simonetti DW, Craig S and Marshak DR: Multilineage potential of adult human mesenchymal stem cells. Science 284: 143-147, 1999.

9. Chen SL, Fang WW, Ye F, Liu YH, Qian J, Shan SJ, Zhang JJ, Chunhua RZ, Liao LM, Lin S and Sun JP: Effect on left ventricular function of intracoronary transplantation of autologous bone marrow mesenchymal stem cell in patients with acute myocardial infarction. Am J Cardiol 94: 92-95, 2004.

10. Wang S, Qu X and Zhao RC: Clinical applications of mesenchymal stem cells. J Hematol Oncol 5: 19, 2012.

11. Hare JM, Fishman JE, Gerstenblith G, DiFede VD, Zambrano JP, Suncion VY, Tracy M, Ghersin E, Johnston PV, Brinker JA, et al: Comparison of allogeneic vs autologous bone marrow-derived mesenchymal stem cells delivered by transendocardial injection in patients with ischemic cardiomyopathy: The POSEIDON randomized trial. JAMA 308: 2369-2379, 2012. 
12. Liang J, Li X, Zhang H, Wang D, Feng X, Wang H, Hua B, Liu B and Sun L: Allogeneic mesenchymal stem cells transplantation in patients with refractory RA. Clin Rheumatol 31: 157-161, 2012.

13. Zhao D, Cui D, Wang B, Tian F, Guo L, Yang L, Liu B and Yu X Treatment of early stage osteonecrosis of the femoral head with autologous implantation of bone marrow-derived and cultured mesenchymal stem cells. Bone 50: 325-330, 2012.

14. Guttman M, Amit I, Garber M, French C, Lin MF, Feldser D, Huarte M,Zuk O, Carey BW, Cassady JP, et al: Chromatin signature reveals over a thousand highly conserved large non-coding RNAs in mammals. Nature 458: 223-227, 2009.

15. Ponting CP, Oliver PL and Reik W: Evolution and functions of long noncoding RNAs. Cell 136: 629-641, 2009.

16. Guttman M, Donaghey J, Carey BW, Garber M, Grenier JK, Munson G, Young G, Lucas AB, Ach R, Bruhn L, et al: lincRNAs act in the circuitry controlling pluripotency and differentiation. Nature 477: 295-300, 2011.

17. Li X, Wu Z, Fu X and Han W: Long noncoding RNAs: Insights from biological features and functions to diseases. Med Res Rev 33: 517-553, 2013.

18. Lee JT and Bartolomei MS: X-inactivation, imprinting, and long noncoding RNAs in health and disease. Cell 152: 1308-1323, 2013.

19. Wang L, Wang Y, Li Z, Li Z and Yu B: Differential expression of long noncoding ribonucleic acids during osteogenic differentiation of human bone marrow mesenchymal stem cells. Int Orthop 39: 1013-1019, 2015.

20. Jiang Y, Jahagirdar BN, Reinhardt RL, Schwartz RE, Keene CD, Ortiz-Gonzalez XR, Reyes M, Lenvik T, Lund T, Blackstad M, et al: Pluripotency of mesenchymal stem cells derived from adult marrow. Nature 418: 41-49, 2002.

21. Livak KJ and Schmittgen TD: Analysis of relative gene expression data using real-time quantitative PCR and the 2(-Delta Delta C(T)) method. Methods 25: 402-408, 2001.

22. Moya-Angeler J, Gianakos AL, Villa JC, Ni A and Lane JM: Current concepts on osteonecrosis of the femoral head. World J Orthop 6: 590-601, 2015.

23. Papakostidis C, Tosounidis TH, Jones E and Giannoudis PV: The role of 'cell therapy' in osteonecrosis of the femoral head. A systematic review of the literature and meta-analysis of 7 studies. Acta Orthop 87: 72-78, 2016.

24. Pepke W, Kasten P, Beckmann NA, Janicki P and Egermann M: Core decompression and autologous bone marrow concentrate for treatment of femoral head osteonecrosis: A randomized prospective study. Orthop Rev (Pavia) 8: 6162, 2016.

25. Mukisi MM, Bashoun K and Burny F: Sickle-cell hip necrosis and intraosseous pressure. Orthop Traumatol Surg Res 95: 134-138, 2009

26. Kumar MN, Belehalli P and Ramachandra P: PET/CT study of temporal variations in blood flow to the femoral head following low-energy fracture of the femoral neck. Orthopedics 37: e $563-\mathrm{e} 570,2014$

27. Zeng X, Zhan K, Zhang L, Zeng D, Yu W, Zhang X, Zhao M, Lai $Z$ and Chen R: The impact of high total cholesterol and high low-density lipoprotein on avascular necrosis of the femoral head in low-energy femoral neck fractures. J Orthop Surg Res 12: 30 , 2017.

28. Jones LC and Hungerford DS: The pathogenesis of osteonecrosis. Instr Course Lect 56: 179-196, 2007.

29. Kerachian MA, Séguin C and Harvey EJ: Glucocorticoids in osteonecrosis of the femoral head: A new understanding of the mechanisms of action. J Steroid Biochem Mol Biol 114: 121-128, 2009.
30. Jilka RL, Noble B and Weinstein RS: Osteocyte apoptosis. Bone 54: 264-271, 2013

31. Wu Z, Ji C, Li H, Qiu G, Gao C and Weng X: Elevated level of membrane microparticles in the disease of steroid-induced vascular osteonecrosis. J Craniofac Surg 24: 1252-1256, 2013.

32. Zheng L, Wang W, Ni J, Li Z and Xiao T: The association of eNOS gene polymorphism with avascular necrosis of femoral head. PLoS One 9: e87583, 2014.

33. Tian L, Wen Q, Dang X, You W, Fan L and Wang K: Immune response associated with Toll-like receptor 4 signaling pathway leads to steroid-induced femoral head osteonecrosis. BMC Musculoskelet Disord 15: 18, 2014.

34. Lee JS, Lee JS, Roh HL, Kim CH, Jung JS and Suh KT: Alterations in the differentiation ability of mesenchymal stem cells in patients with nontraumatic osteonecrosis of the femoral head: Comparative analysis according to the risk factor. J Orthop Res 24: 604-609, 2006.

35. Huang S, Wang S, Bian C, Yang Z, Zhou H, Zeng Y, Li H, Han Q and Zhao RC: Upregulation of miR-22 promotes osteogenic differentiation and inhibits adipogenic differentiation of human adipose tissue-derived mesenchymal stem cells by repressing HDAC6 protein expression. Stem Cells Dev 21: 2531-2540, 2012.

36. Zeng Y, Qu X, Li H, Huang S, Wang S, Xu Q, Lin R, Han Q, Li J and Zhao RC: MicroRNA-100 regulates osteogenic differentiation of human adipose-derived mesenchymal stem cells by targeting BMPR2. FEBS Lett 586: 2375-2381, 2012.

37. Wang Q, Yang Q, Chen G, Du Z, Ren M, Wang A, Zhao H, Li Z, Zhang $\mathrm{G}$ and Song Y: LncRNA expression profiling of BMSCs in osteonecrosis of the femoral head associated with increased adipogenic and decreased osteogenic differentiation. Sci Rep 8: 9127,2018

38. Wu D, Xu Y, Zou Y, Zuo Q, Huang S, Wang S, Lu X, He X, Wang J, Wang T and Sun L: Long noncoding RNA 00473 is involved in preeclampsia by LSD1 binding-regulated TFPI2 transcription in trophoblast cells. Mol Ther Nucleic Acids 12: 381-392, 2018.

39. Wang L, Zhang X, Sheng L, Qiu C and Luo R: LINC00473 promotes the Taxol resistance via miR-15a in colorectal cancer. Biosci Rep 38: BSR20180790, 2018.

40. Zhang W and Song Y: LINC00473 predicts poor prognosis and regulates cell migration and invasion in gastric cancer. Biomed Pharmacother 107: 1-6, 2018.

41. Wang D, Li J, Cai F, Xu Z, Li L, Zhu H, Liu W, Xu Q, Cao J, Sun J and Tang J: Overexpression of MAPT-AS1 is associated with better patient survival in breast cancer. Biochem Cell Biol 97: 158-164, 2019.

42. Pan Y, Pan Y, Cheng Y, Yang F, Yao Z and Wang O: Knockdown of LncRNA MAPT-AS1 inhibites proliferation and migration and sensitizes cancer cells to paclitaxel by regulating MAPT expression in ER-negative breast cancers. Cell Biosci 8: 7, 2018.

43. Coupland KG, Kim WS, Halliday GM, Hallupp M, Dobson-Stone C and Kwok JB: Role of the long non-coding RNA MAPT-AS1 in regulation of microtubule associated protein Tau (MAPT) expression in Parkinson's disease. PLoS One 11: e0157924, 2016.

This work is licensed under a Creative Commons Attribution-NonCommercial-NoDerivatives 4.0 International (CC BY-NC-ND 4.0) License. 